\title{
Das ausbildungsintegrierende duale Studium
}

Das duale Studium gewinnt in der bildungspolitischen Landschaft in Deutschland zunehmend an Bedeutung. Keine Abi-Messe, die diesen Bildungsweg nicht an hervorgehobener Stelle bewirbt. Forciert wird diese Entwicklung zum einen durch das veränderte Bildungsverhalten der studienberechtigten Jugendlichen sowie zum anderen durch die Personalpolitik der Betriebe. Sie ist auf Nachwuchssicherung qualifizierter Schulabgänger und eine damit verbundene frühzeitige Fachkräftebindung gerichtet. Die ausbildungsintegrierende Form des dualen Studiums kombiniert eine berufliche Ausbildung mit einem berufsqualifizierenden Studium. Welchen Stellenwert hat aber die berufliche Ausbildung in diesem Studium? Und werden die Absolventen dieser Studiengänge auf die Stellen drängen, die bislang von Facharbeitern und Kaufleuten eingenommen werden?

SIRIKIT KRONE, ULRICH MILL

\section{Problemstellung}

Duale Studiengänge haben sich in Deutschland etabliert und sind weiterhin auf Expansionskurs. In der Erstausbildung ermöglichen sie jungen Menschen mit (Fach)Hochschulreife die Möglichkeit, parallel zwei Abschlüsse zu erlangen: einen beruflichen im dualen Ausbildungssystem sowie einen akademischen an der Hochschule. Diese Doppelqualifikation im dualen Studium ist für viele studienberechtigte Jugendliche sehr attraktiv. Der hohe Praxisbezug und die guten Karrierechancen sind die vorrangigen Motive für Schulabgänger, sich für diese Studienform, die ein hohes Maß an Disziplin und Selbstorganisation von ihnen verlangt, zu entscheiden (Krone/Mill 2012; Kupfer et al. 2012). Der steigenden Nachfrage steht zwischenzeitlich ein relativ breit gefächertes Angebot an den Hochschulen gegenüber. Das Konzept des dualen Studiums basiert auf der Verknüpfung zweier bisher starr voneinander getrennter Bildungssegmente, und die Entwicklung ist eingebettet in die bildungspolitischen Debatten um Öffnung der Hochschulen für neue Zielgruppen, Gleichwertigkeit beruflicher und akademischer Ausbildung sowie Outcome-Orientierung der Lernprozesse.

In unserem Beitrag gehen wir der Frage nach, welchen Stellenwert die berufliche Ausbildung in hybriden Ausbildungsformen einnimmt - hier am Beispiel des dualen Studiums. ${ }^{1}$ Wir gehen von der Annahme aus, dass die
Verknüpfung beruflicher und akademischer Ausbildung eine zentrale Bedeutung für den betrieblichen Einsatz der Absolventen dualer Studiengänge hat, und fragen nach den Motiven der Unternehmen, sich an dualen Studienmodellen zu beteiligen. In der Diskussion hält sich die These eines Verdrängungswettbewerbs von Absolventen beruflicher Ausbildungs- und Aufstiegswege mit solchen, die über einen akademischen Abschluss verfügen. Diese Frage wird, wie wir weiter unten ausführen, in der Literatur unterschiedlich beantwortet. Wir erweitern sie um eine zusätzliche Komponente: Hat sich eine Konkurrenzsituation zwischen den Absolventen klassischer, betrieblicher wie akademischer Ausbildungswege und denen dualer Studiengänge entwickelt, die zu Verdrängungseffekten im betrieblichen Personaleinsatz führt?

Zur Einführung des wenig bekannten Forschungsgegenstandes wird im Folgenden zunächst ein Überblick über die verschiedenen Modelle und Konzeptionen dualer Studiengänge gegeben (Abschnitt 2). Die Entwicklung klassischer Ausbildungs- und Aufstiegswege liefert den Begründungszusammenhang für die Entstehung und den Ausbau hybrider Konzepte wie das duale Studium (3). Wir formulieren drei zentrale Faktoren, die den Trend in den

1 Vgl. Graf 2012 zu Begriff und Entwicklung hybrider Ausbildungsformen. 
TABELLE 1

\section{Angebot dualer Studiengänge in Deutschland}

\begin{tabular}{c|c|c|c}
\hline & $\begin{array}{c}\text { Anzahl dualer } \\
\text { Studiengänge }\end{array}$ & $\begin{array}{c}\text { Beteiligte } \\
\text { Unternehmen }\end{array}$ & Studienplätze \\
\hline 2005 & 545 & 18.911 & 42.467 \\
\hline 2011 & 929 & 40.874 & 61.195 \\
\hline
\end{tabular}

* Im Jahr 2012 wurden erstmals duale Studiengänge zur Weiterbildung in großem Umfang in die Datenbank AusbidungPlus aufgenommen, weshalb ein großem Umang in die Datenbank AusbidungPlus aufgenommen, weshalb ein Vergle mit diesen aktuellen Da die Zuverlässigkeit der Datenbank AusbildungPlus eingeschränkt, da die dort aufgenommenen Daten auf freiwilliger Selbstmeldung der Anbieter beruhen.

Quelle: Datenbank AusbildungPlus*

vergangenen Jahren angetrieben haben: die deutsche Bildungspolitik, das veränderte Bildungsverhalten der studienberechtigten Jugendlichen sowie die Personalpolitik der Betriebe. Als Antwort auf die veränderte Nachfrage sowohl aufseiten der Schulabgänger als auch aufseiten der Betriebe nach neuen, integrierten Angeboten stellen wir das duale Studium aus der Perspektive der nachfragenden Akteure vor. Motive und Einschätzungen der Studierenden wie der beteiligten Unternehmen werden anhand der Ergebnisse unserer empirischen Forschungsarbeiten im Rahmen eines zwischen 2010 und 2013 durchgeführten Projektes dargestellt. ${ }^{2}$ Sie erlauben uns Aussagen zur Bedeutung der Verknüpfung beruflicher und akademischer Ausbildung für den betrieblichen Einsatz der Absolventen dualer Studiengänge sowie zu den Motiven der beteiligten Akteure.

Die Frage, ob es zu einem Verdrängungswettbewerb zwischen Absolventen klassischer Ausbildungswege und denen aus dem dualen Studium kommt, beantworten auch wir auf der Grundlage unserer Empirie widersprüchlich (4). Schließlich lenken wir den Blick auf die Rolle der betrieblichen Ausbildung in einem Studium, das sich die Integration der beruflichen Ausbildung auf die Fahne geschrieben hat (5). Der Beitrag schließt mit Fazit und Ausblick auf eine zukünftige Untersuchung zum Einsatz der Absolventen dualer Studiengänge (6).

\section{Modelle dualer Studiengänge}

Das duale Studium findet vorwiegend an Fachhochschulen statt: Ca. 60 \% der Studiengänge werden dort angeboten. Universitäten beteiligen sich nur in Einzelfällen an dieser Form der Ausbildung. Vermehrt haben auch private Hochschulen diesen Markt für sich entdeckt und platzieren sich bundesweit mit einem umfangreichen Angebot. Ihre höhere Flexibilität und Bereitschaft, sich auf die jeweiligen Bedarfe der kooperierenden Betriebe einzustellen, macht sie als Kooperationspartner attraktiv. Sie nutzen die Chance, ihr Profil mit diesem spezifischen Angebot zu schärfen und sich neuen Zielgruppen gegenüber zu öffnen.

Politisch wird der Ausbau dualer Studienangebote in einigen Bundesländern stark forciert und teilweise gezielt mit Fördermitteln unterstützt, insbesondere in NordrheinWestfalen, Baden-Württemberg und Bayern. ${ }^{3}$ Nicht zuletzt deshalb ist bundesweit eine deutliche Expansion zu verzeichnen - von 2005 bis 2011 ein Zuwachs von ca. $70 \%$. Parallel dazu ist die Zahl der Studierenden sowie der beteiligten Betriebe ebenfalls gewachsen, woran das zunehmende Interesse auf beiden Seiten deutlich wird (Tabelle 1).

Fachlich liegt der Schwerpunkt mit einem Anteil von gut Zweidritteln beim Angebot dualer Studiengänge in den Ingenieur- und Wirtschaftswissenschaften, wobei das Fächerspektrum in den vergangenen Jahren deutlich vielfältiger geworden ist und mittlerweile auch gesundheitsund pflegewissenschaftliche oder frühpädagogische Studiengänge einschließt.

Es existiert eine Vielzahl verschiedener Konzepte dualer Studiengänge, die in vier Formen systematisiert werden können (Tabelle 2), zwei angelegt als Erstausbildung und zwei als Weiterbildung, wobei die deutliche Mehrzahl der Studiengänge der Erstausbildung zuzuordnen ist. ${ }^{4}$

Im Folgenden beschränken wir uns auf die ausbildungsintegrierende Variante, da nur hier die in Ausbildungsordnungen institutionalisierte Berufsausbildung abschlussorientiert integriert wird. In Abhängigkeit von den unterschiedlichen rechtlichen Vorschriften in den einzelnen Bundesländern ist hier teilweise - neben den Lernorten Betrieb und Hochschule - auch die Berufsschule als dritter Lernort involviert. Als Zugangsvoraussetzung zu
2 Die spärliche Forschungslage zum dualen Studium motivierte uns zu einem vom Bundesministerium für Bildung und Forschung (BMBF) geförderten Forschungsprojekt, das am Institut Arbeit und Qualifikation (IAQ) im Zeitraum 10/2012 bis 3/2013 unter dem Titel „Duale Studiengänge Entstehungsbedingungen, Interessenlagen und Umsetzungserfahrungen" durchgeführt wurde (www.iaq.unidue.de/dual/index.php). Unsere Untersuchung umfasst 20 Fallstudien ausbildungsintegrierender dualer Studiengänge in ingenieurwissenschaftlichen und wirtschaftswissenschaftlichen Fachrichtungen. Dabei wurden 104 qualitative Interviews mit Hochschul-, Unternehmens-, Berufsschulund Kammervertretern geführt. Die Positionen der Studie- renden aus den untersuchten Studiengängen wurden in einer teilstandardisierten Online-Befragung ermittelt (Rücklauf $n=485$ ), ergänzt durch 15 Gruppendiskussionen.

3 Die jüngste Initiative ist im August 2013 unter Beteiligung der drei Ministerien für Wirtschaft, Wissenschaft und Kultur in Hessen gestartet worden; www.dualesstudium-hessen.de/presse/news/.

4 Von den im Jahr 2012 in der Datenbank AusbildungPlus gelisteten 1.384 Angeboten dualer Studiengänge bezogen sich 910 auf die berufliche Erstausbildung,

vgl. http://www.ausbildungplus.de. 
Systematisierung der Modelle dualer Studiengänge

\begin{tabular}{|c|c|c|c|c|}
\hline & $\begin{array}{l}\text { ausbildungs- } \\
\text { integrierend }\end{array}$ & praxisintegrierend & berufsintegrierend & berufsbegleitend \\
\hline Bildungsstufe & \multicolumn{2}{|c|}{ Erstausbildung } & \multicolumn{2}{|c|}{ Weiterbildung } \\
\hline $\begin{array}{c}\text { Zugangs- } \\
\text { qualifikation }\end{array}$ & $\begin{array}{c}\text { (Fach) } \\
\text { Hochschulreife }\end{array}$ & $\begin{array}{c}\text { (Fach) } \\
\text { Hochschulreife }\end{array}$ & $\begin{array}{l}\text { Berufsausbildung } \\
\text { und Berufserfahrung }\end{array}$ & $\begin{array}{l}\text { (Fach)Hochschulreife } \\
\text { und ggf. } \\
\text { Berufsausbildung / } \\
\text {-erfahrung }\end{array}$ \\
\hline $\begin{array}{l}\text { betriebliche } \\
\text { Integration }\end{array}$ & $\begin{array}{l}\text { Ausbildungs- oder } \\
\text { Praktikantenvertrag }\end{array}$ & $\begin{array}{l}\text { Praktikanten-, } \\
\text { Volontariats- } \\
\text { oder (Teilzeit) } \\
\text { Arbeitsvertrag }\end{array}$ & $\begin{array}{l}\text { (Teilzeit) } \\
\text { Arbeitsvertrag } \\
\text { (Betrieb gewährt } \\
\text { Freistellungen) }\end{array}$ & $\begin{array}{l}\text { Arbeitsvertrag } \\
\text { (Betrieb gewährt } \\
\text { Freistellungen) }\end{array}$ \\
\hline $\begin{array}{l}\text { curriculares } \\
\text { Konzept }\end{array}$ & $\begin{array}{l}\text { inhaltliche und zeitliche } \\
\text { Verzahnung von Aus- } \\
\text { bildung und Studium }\end{array}$ & $\begin{array}{l}\text { inhaltliche Bezüge } \\
\text { zwischen betrieblicher } \\
\text { Praxis und Studium }\end{array}$ & $\begin{array}{l}\text { inhaltliche Bezüge } \\
\text { zwischen betrieblicher } \\
\text { Praxis und Studium }\end{array}$ & Hochschulstudium \\
\hline $\begin{array}{l}\text { Bildungs- } \\
\text { abschluss }\end{array}$ & $\begin{array}{l}\text { Ausbildungsberuf, } \\
\text { Bachelor } \\
\text { bzw. Master }\end{array}$ & Bachelor & $\begin{array}{l}\text { Bachelor und ggf. } \\
\text { Weiterbildungs- } \\
\text { abschluss }\end{array}$ & $\begin{array}{c}\text { Bachelor } \\
\text { bzw. Master }\end{array}$ \\
\hline $\begin{array}{l}\text { Ausbildungs- bzw. } \\
\text { Studienzeit }\end{array}$ & 3-5 Jahre & 3 Jahre & i.d.R. 3 Jahre & 3-5 Jahre \\
\hline
\end{tabular}

Quelle: Datenbank AusbildungPlus; Darstellung der Autoren.

einem dualen Studium benötigen die Bewerber über die Hochschulzugangsberechtigung hinaus zunächst einen Ausbildungs- bzw. Praktikantenvertrag mit einem kooperierenden Betrieb, der die betriebliche Ausbildung übernimmt. Damit kommt den Unternehmen eine besondere Gatekeeper-Funktion an der Schnittstelle zwischen Abitur und Studium zu. Nicht mehr das Bildungssystem allein (etwa über einen Numerus clausus) regelt den Zugang, sondern dieser ist abhängig von dezentralen Entscheidungen privater Unternehmen. Den betrieblichen Mitbestimmungsgremien kommt dabei eine wichtige Funktion bei der Entwicklung der Auswahlkriterien sowie im Einstellungsverfahren zu.

\section{Entwicklungsbedingungen des Aus- baus dualer Studiengänge}

Die sich wechselseitig bedingenden Entwicklungen des Bildungsverhaltens studienberechtigter Jugendlicher und der Personalpolitik in den Betrieben bei der Nachwuchsrekrutierung führen zu einer veränderten Nachfrage am Ausbildungsmarkt. Ein Trend hin zur Akademisierung der Bildungswege zulasten klassischer Berufsbildungswege ist unübersehbar, wie die dramatische Entwicklung der Studienanfängerquoten pro Geburtsjahrgang von 31,3\% im Jahr 1999 auf 54,0 \% im Jahr 2011 zeigt (Statistisches Bundesamt 2012, S. 11). Daraus folgen Anreize für Bildungsanbieter, sich auf den neuen Bedarf einzustellen und adäquate Angebote zu entwickeln. Das duale Studium ist ein solches
Angebot; es stellt eine Reaktion auf zwei zentrale Trends am Berufsbildungsmarkt dar: die sinkende, aber trotzdem immer noch hohe Relevanz der dualen Berufsausbildung sowie der Trend hin zur Akademisierung von Bildungswegen. Im Wesentlichen sehen wir im hier diskutierten Kontext drei relevante Faktoren, welche diese Entwicklung in den vergangenen Jahren bedingt haben.

\subsection{Deutsche Bildungspolitik}

Im Zuge der Entstehung eines europäischen Bildungsraumes, inhaltlich gestaltet durch die Erklärungen von Bologna (1999) und Kopenhagen (2002), entwickelte sich in Deutschland eine Debatte um die Relevanz der Akademisierung des deutschen Berufsbildungssystems nach europäischem Vorbild (z. B. Baethge et al. 2007; Kruse et al. 2009; Kuda/Strauß 2010a, 2010b, 2011; Strauß/Kuda 2010). Im internationalen Vergleich ist die Studierendenquote in Deutschland nach wie vor relativ gering, wie an den jährlich veröffentlichten Zahlen der Organisation für wirtschaftliche Zusammenarbeit und Entwicklung (OECD 2012, 2013) deutlich wird. Daraus wurde auf Bundesebene in der Bildungspolitik der letzten Jahre der Schluss gezogen, dass ein Ausbau des Tertiärsystems notwendig ist, mit dem Ziel, im europäischen Bildungsraum konkurrenzfähig zu bleiben (vgl. zu aktuellen berufsbildungspolitischen Maßnahmen und Programmen BMBF 2012, S. 4ff.; 2013, S. 53ff.).

Allerdings wird die Notwendigkeit einer Angleichung der Studierendenquote an das europäische Niveau durchaus unterschiedlich beurteilt (z. B. Bosch 2012). Kritische Stimmen verweisen auf die Tatsache, dass das System der beruf- 
lichen Bildung in Deutschland auf einem qualifikatorisch sehr hohen Niveau entwickelt sei, welches dem akademischen in Teilen durchaus vergleichbar sei. Gerade in dieser Gleichwertigkeit bei Andersartigkeit der Abschlüsse der beruflichen Aus- und Aufstiegsfortbildung auf der einen und den im tertiären Sektor erworbenen auf der anderen Seite liegt die Problematik der Vergleichbarkeit und gegenseitigen Anerkennung (Klumpp et al. 2010; Weigel et al. 2011). Die lange Debatte um die Einstufung der Qualifikationen und die damit verbundene Wertigkeit der Bildungsabschlüsse in der Umsetzung des europäischen Qualifikationsrahmens in den nationalen deutschen Qualifikationsrahmen zeigen diesen Konflikt deutlich (Nehls in diesem Heft). Insbesondere die in diesem Kontext heftig geführte Diskussion um Gleichwertigkeit beruflicher und akademischer Bildung und der daraus folgenden Einstufung der beruflichen Aufstiegsabschlüsse Meister, Techniker, Fachwirt auf dem Bachelorniveau trägt dem deutschen Bildungssystem und seinen Spezifika Rechnung.

\subsection{Bildungsverhalten studienberechtigter Jugendlicher}

Parallel zur bildungspolitischen Diskussion um die Erhöhung der Studierendenquote hat sich das Bildungsverhalten derjenigen Jugendlichen, die die Schule mit dem Abitur bzw. Fachabitur verlassen, deutlich gewandelt. Auch hier ist ein Trend zur Abwanderung in den tertiären Bereich mit dem Ziel erkennbar, die eigenen Chancen auf dem Arbeitsmarkt zu erhöhen. Bereits im sekundären Bildungssektor ist eine Entwicklung hin zu höheren Abschlüssen zu beobachten: Die Zahl der Schulabgänger mit Hochschulzugangsberechtigung nimmt kontinuierlich zu; zwischen 2001 und 2011 stieg „die Studienberechtigtenquote um knapp 21 Prozentpunkte" (Statistisches Bundesamt 2013, S. 9).

Höhere schulische Abschlüsse gelten als Voraussetzung für einen erfolgreichen Einstieg in den Ausbildungs- und Arbeitsmarkt. Mit dieser Entwicklung geht ein Attraktivitätsverlust der beruflichen Bildung bei Schulabgängern und ihren Eltern einher, wenn es um die Planung ihrer beruflichen Zukunft geht. Es dominiert die Überzeugung, dass ein akademischer Abschluss notwendig ist, um beruflich Karriere zu machen. Auch die von uns im Rahmen eines Forschungsprojektes befragten dual Studierenden verfolgten eine klare akademische Orientierung: Gefragt nach ihrer Alternative zum dualen Studium nannten 76,1\% die Aufnahme eines Vollzeitstudiums. Die Möglichkeit, „zwei Abschlüsse“ zu erwerben, ist für $81,9 \%$ der von uns befragten Studierenden ein Motiv für die Aufnahme eines dualen Studiums, der erwartete „hohe Praxisbezug des Studiums“ (im Vergleich zu einem Vollzeitregelstudium) ist dies für 94,6\% von ihnen. Nach erfolgreichem Abschluss erwarten 88,4\% von ihnen einen sicheren Einstieg im Ausbildungsbetrieb bereits auf der mittleren Führungsebene mit der Perspektive eines schnellen Aufstiegs, der für $81 \%$ der Befragten ein Motiv zur Aufnahme des dualen Studiums darstellt. Die Unternehmen reagieren auf diese Erwartungen sehr gut qualifizierter Schulabgänger mit dem Angebot neu definierter Karrierewege. Damit bleiben sie als Ausbildungsbetrieb für diese hoch qualifizierte und motivierte Klientel attraktiv. Bereits während der Ausbildung werden qualifizierte Einsatzfelder für die dual Studierenden in den Betrieben definiert, in denen sie anspruchsvolle Aufgaben erledigen.

\subsection{Personalpolitik der Betriebe}

Unternehmen vieler Branchen haben in zunehmendem Maße Schwierigkeiten, adäquat qualifizierte Schulabgänger für ihre Ausbildungsplätze im höheren Segment zu finden. Da die Mehrzahl der Abiturienten ein Studium anstrebt, fehlt diese Gruppe für die klassischen Karrierewege der betrieblichen Ausbildung und Aufstiegsfortbildung. Die Autoren der Qualifikations- und Berufsfeldprojektionen des Bundesinstituts für Berufsbildung (BIBB) und des Instituts für Arbeitsmarkt- und Berufsforschung (IAB) gehen bis zum Jahr 2030 davon aus, dass „[...] die sich im akademischen Bereich abzeichnende neue Bildungsexpansion [...] rein quantitativ zu einem Mangel an Fachkräften auf der mittleren Qualifikationsebene in einigen Berufsfeldern führen“ (Helmrich et al. 2012, S. 2) könnte. Insofern sind viele Unternehmen gezwungen, neue Wege der Rekrutierung qualifizierten Nachwuchses zu gehen.

Das Motiv der Fachkräftegewinnung ${ }^{5}$ bzw. ihre frühzeitige Bindung an den Betrieb ist dementsprechend das Leitmotiv für Unternehmen, sich an der Entwicklung, Implementation und Durchführung dualer Studiengänge zu beteiligen, auf das sich, neben Ressourcensicherung und -ausbau sowie Konkurrenzvorteilen auf dem Bildungsmarkt, auch die Hochschulen beziehen. Vorrangig mittlere bis größere Unternehmen nutzen duale Studiengänge bisher für ihren Führungskräftenachwuchs, wobei jedoch auch Betriebe des Handwerks mehr und mehr im Rahmen von Kammerinitiativen ein duales Studium anbieten. So finden sich unter den Anbietern dualer Studiengänge sowohl Mittelständler als auch die Spitzenunternehmen der deutschen Wirtschaft. Unternehmen entsprechen mit ihrer Beteiligung an dualen Studiengängen dem steigenden Fachkräftebedarf sowie den verbreiterten und teilweise gestiegenen Anforderungen in wissensbasierten Berufen und binden so qualifizierte Schulabgänger an ihren Betrieb. Sie decken mit den Absolventen der doppelqualifizierenden Ausbildungsgänge ihren Bedarf an akademisch vorgebildeten Führungskräften mit hoher Praxisorientierung ab. Ein typisches Beispiel aus unserem Projekt:

„Dieses ausbildungsintegrierende Studium ist für uns ganz automatisch gekommen, als ich festgestellt habe, dass die guten Bewerber, die also ein gutes Abitur haben,

5 „Die Fachkräfte umfassen sowohl Personen mit einer anerkannten akademischen als auch einer anerkannten mindestens zweijährigen Berufsausbildung" (Helmrich et al. 2012, S. 11) 
nicht mehr nur eine Ausbildung machen wollen, [...] wenn der Markt das will, dann nutzen wir das auch. Also das ist eben unser zweites Standbein [...], weil die hochleistungsfähigen, 19-jährigen Abiturienten darüber eben auch an unser Haus gebunden werden." (Fall I, Betrieb B, \$12)

Unternehmen erhalten über dieses Ausbildungssegment hochqualifizierte und langfristig gebundene Mitarbeiter, die aufgrund der Dualität der Berufsbilder und der Lernorganisationen eine besondere Innovationsfähigkeit in die vorhandenen betrieblichen Arbeits- und Organisationsstrukturen einbringen können. Für die Mehrzahl der Unternehmen stehen neben der Kombination praktischer und theoretischer Kompetenzen insbesondere die kommunikativen Fähigkeiten der Absolventen dualer Studiengänge in Richtung Produktionsebene einerseits und Leitungsebene andererseits im Vordergrund. In der Konkurrenz um die klügsten Köpfe bietet das duale Studium gerade für Betriebe an unattraktiven Standorten die Möglichkeit, sich für diese Zielgruppe als interessanter Arbeitgeber bereits frühzeitig zu profilieren und damit junge Menschen in der Region zu halten und für den eigenen betrieblichen Bedarf passgenau auszubilden.

\section{Verdrängungswettbewerb?}

Das wachsende Angebot von Absolventen dualer Studiengänge legt die Vermutung nahe, dass sich hier ein Wettbewerb zwischen den Absolventen verschiedener Ausbildungswege entwickelt und es zu einer Verdrängung der Bewerber mit klassischen Abschlüssen kommt. Diese Debatte wird bereits seit Längerem in Bezug auf mögliche Konkurrenzen zwischen Bachelorabsolventen und solchen aus der dualen Berufsausbildung mit Abschlüssen der Aufstiegsfortbildung geführt. Unternehmen reagieren bei ihren Einstellungsentscheidungen positiv auf das wachsende Angebot von Bewerbern mit Hochschulabschluss. Insofern stellt sich die Frage, inwiefern sich auf der mittleren Führungsebene die Facharbeiter mit einer Aufstiegsfortbildung mittelfristig in einem Verdrängungswettbewerb gegenüber den Bachelorabsolventen behaupten müssen. Hierzu liegen unterschiedliche Ergebnisse aus empirischen Forschungen der letzten Jahre vor.

So konstatiert Drexel (2012), dass es zu einem Verlust an Aufstiegsmöglichkeiten für Absolventen des dualen Systems kommt und Betriebe Positionen im mittleren Management verstärkt mit Akademikern besetzen. Begleitet wird dieser Prozess mit einer Neustrukturierung der Tätigkeitsfelder dieser Positionen von einer stärkeren Polarisierung zwischen den Arbeitsplätzen der Akademikerebene (welche dann für die Aufsteiger aus der Aufstiegsfortbildung verschlossen bleiben) auf der einen und der Ebene der einfachen Tätigkeiten auf der anderen Seite sowie einer tendenziellen Minimierung der Tätigkeiten auf der mittleren Ebene.
Franz und Voss-Dahm (2011) zeigen in einer Analyse von Daten des Sozio-oekonomischen Panels von 2008 auf, dass zwei Drittel der deutschen Führungskräfte über einen akademischen Abschluss verfügen, jede Zweite hat allerdings zuvor (auch) eine Ausbildung im beruflichen Bildungssystem absolviert. Die Forscherinnen konstatieren, dass sich die Konkurrenz zwischen beruflicher und akademischer Bildung nicht verschärft hat, sondern der Anteil derjenigen Führungskräfte, die eine Doppelqualifikation besitzen, zunimmt. Betriebe nutzen also die Kompetenzen aus beiden Bildungssegmenten.

$\mathrm{Zu}$ einer ähnlichen Einschätzung kommen eine Forschungsgruppe aus dem BIBB (Wünsche 2011) sowie die Autoren einer Studie des Instituts der deutschen Wirtschaft (Hollmann et al. 2010). Nach den Ergebnissen der BIBBForschungsgruppe führt die Zunahme an Akademikern nicht zu einer Verdrängung beruflich Qualifizierter. Aus den Ergebnissen ihrer Betriebsfallstudien schließen die $\mathrm{Au}$ toren, dass die Unternehmen vielmehr versuchen, die jeweils spezifischen Vorteile akademischer und beruflichbetrieblicher Ausbildung zu verknüpfen. Hollmann et al. (2010) erkennen in den Unternehmen parallele Laufbahnen und Tätigkeitsfelder für beruflich und akademisch ausgebildetes Personal, wobei die Tätigkeitsfelder der qualifizierten Facharbeiter am oberen Rand des Leistungsspektrums näher an das Hochschulsystem heranrücken. Hier sehen sie durchaus Überschneidungen in den Tätigkeitsprofilen.

In der empirischen Forschung bisher weitgehend unbeantwortet bleibt die Frage, wie die Bewerber mit ihren spezifischen, im dualen Studium erworbenen, Bildungssegmente übergreifenden Abschlüssen in die Personalplanung der Unternehmen integriert werden und welche spezifischen Karrierewege für sie vorgesehen sind. In der Diskussion hält sich auch hier die These des Verdrängungswettbewerbs, im Zuge dessen die Absolventen dualer Studiengänge Positionen besetzen, die zuvor reine Akademiker oder Aufsteiger über klassische Fortbildungswege einnahmen. Die wenigen empirisch ausgerichteten Arbeiten, die sich mit der direkten Verbindung von beruflicher und Hochschulbildung sowie möglichen Konsequenzen für die Karrierepfade in den Unternehmen beschäftigen (z. B. Kloas 2007; Werner et al. 2008; Klumpp/Rybnikova 2010; Heidemann 2011; Purz 2011), kommen zu teilweise widersprüchlichen Ergebnissen. Während einige Analysen auf der Grundlage von Fallstudien bzw. Interviews mit Unternehmensvertretern Konkurrenzen aufzeigen, beschreiben andere das duale Studium als einen parallel zu den anderen verlaufenden Ausbildungs- und Karrierepfad.

Unsere Empirie bestätigt diese Widersprüche. Die Mehrzahl der von uns befragten Unternehmensvertreter berichtet, dass sie die Absolventen dualer Studiengänge eher als eine zusätzliche Option für ihre Personaleinsatzplanung sieht, für die sie teilweise eigene Karrierepfade mit einem höheren Einstiegsniveau als solche für Bachelorabsolventen entwickelt. Dies ist insbesondere vor dem Hintergrund zu 
sehen, dass die Investitionskosten für den Betrieb im dualen Studium relativ hoch sind und die Unternehmen ein großes Interesse haben, diese Auszubildenden zu übernehmen. Dabei werden zwei zentrale Erfolgsfaktoren der dualen Berufsausbildung auf das tertiäre Niveau übertragen: Verankerung der Auszubildenden bzw. Studierenden in fachlichen Abläufen sowie Sozialstrukturen im Betrieb und damit verbunden eine hohe Integrationsfunktion beim Übergang in den Arbeitsmarkt. Einige Unternehmen, die Absolventen aus dem wirtschaftswissenschaftlichen Bereich rekrutieren, sahen allerdings sehr wohl eine Konkurrenzsituation zwischen den Absolventen klassischer Ausbildungswege und denen aus dem dualen Studium. Beispielsweise im Bankbereich konkurrieren sowohl die Absolventen der Aufstiegsfortbildungen (Bankfachwirt, Bankbetriebswirt) als auch die dualen und die einfachen Bachelorabsolventen um die gehobenen Aufgaben (im Privatkunden-, Geschäftskunden-, Immobilienbereich) und Führungspositionen. Die Konkurrenz entsteht, so unsere Hypothese auf der Grundlage von Interviews mit acht Banken und einem Bankenverbund, weil die Karrierepfade nicht getrennt sind. Ob diese Hypothese sich bewährt, könnte erst eine breit angelegte vergleichende Untersuchung der Karrierepfade dieser Absolventen unterschiedlicher Bildungsgänge ergeben.

\section{Die Rolle der betrieblichen Berufs- ausbildung}

Die Übertragung der Erfolgsfaktoren der dualen Berufsausbildung auf das duale Studium scheint deren Absolventen einen komparativen Wettbewerbsvorteil zu bieten. Wir wollen an dieser Stelle etwas genauer auf die Rolle der betrieblichen Ausbildung im ausbildungsintegrierenden dualen Studium eingehen.

Würde auf eine normale duale Berufsausbildung, die drei oder dreieinhalb Jahre dauert, ein Bachelorstudium folgen, das ebenfalls drei bis dreieinhalb Jahre dauert, dann hätte man ein sechs- bis siebenjähriges duales Studium. Tatsächlich beträgt die Regelstudienzeit (inklusive Ausbildungszeit) für ein duales Studium drei bis fünf Jahre (für den Bachelorabschluss). Es müssen also, sollen diese Vorgaben eingehalten werden, Ausbildungs- und Studienanteile gekürzt werden. Beim Studium an der Hochschule sind dies in der Regel im Curriculum des Studiums verankerte Praxisanteile. Außerdem wird die gesamte Ausbildungs- und Studienzeit intensiv genutzt - Semesterferien z. B. sind für dual Studierende nicht vorgesehen, in manchen Studiengängen finden auch am Samstag Veranstaltungen in den Hochschulen statt. Bei einem dualen Studiengang mit insgesamt vier Jahren Ausbildung und Studium heißt es: „In den ersten zwei Jahren parallel zum Studium eine Berufsausbildung in einem an diesem Studiengang beteiligten Unternehmen, mit abschließender IHK-Prüfung" (Fallstudie J, Posterpräsentation). Die
Ausbildungszeit wurde in allen untersuchten dualen Studiengängen deutlich gekürzt. „[...] wir machen den Stoff der Zwischenprüfung, da braucht ein normaler Industriekaufmann 20 Blockwochen und wir machen das jetzt in 11 Blockwochen“" (Fall T, Studierender/Gruppendiskussion, \$183). Ein Ausbildungsleiter eines an einem dualen Studiengang beteiligten Unternehmen rechnete vor: „Das heißt, dass sie (die dual Studierenden) hochgerechnet in einer normalerweise 2,5 oder 3,5 Jahre dauernden Ausbildung zum Fluggerätemechaniker nur insgesamt 5 - 6 Monate im Unternehmen sind.“ (Fallstudie H, Unternehmen 3, \$66). Bei einem anderen Studiengang ist ein Mindestausbildungszeitraum von 60 Wochen vereinbart worden (Fallstudie V) und in einem Studiengang an einer Fachhochschule, die ein auch von anderen dualen Studiengängen übernommenes Modell entwickelt hat, geht man von einer eindreivierteljährigen Ausbildungszeit aus (Fallstudie L).

Typischerweise wurde die Verkürzung der Ausbildungszeit in unseren Interviews und in den Gruppendiskussionen mit den Studierenden unter dem Blickwinkel der starken Arbeitsbelastung thematisiert. Nicht wenige Stimmen bezogen sich aber auch kritisch auf das Verhältnis von Input (Ausbildungsumfang) und Outcome (fachliche Handlungskompetenz), wie der bereits zitierte Ausbildungsleiter: „Es fehlt (die Zeit) das Gelernte zu konsolidieren."(Fallstudie H, Unternehmen 3, §68).

Ähnlich argumentiert ein Dual-Studierender: „Aber gerade jetzt z. B. in meinem Unternehmen würde ich eher sagen, dass halt auch dieser Praxisanteil, den wir halt in der Ausbildung noch mit haben, gar nicht so sehr dazu ausreicht, um Facharbeitern wirklich das Wasser reichen zu können. Weil er ja doch schon eher mit seinem Abschluss fertig ist, schon länger damit quasi arbeitet, wenn er jetzt mit 16 die Ausbildung angefangen hat und er hat da schon einen gewissen Vorsprung, denke ich mal.“(Dual-Studierender in einer Gruppendiskussion, Fallstudie B, §72)

In beiden Stellungnahmen geht es darum, dass zur fachlichen Handlungskompetenz von Facharbeiterinnen und Facharbeitern wesentlich der Aufbau von Erfahrung gehört - und Erfahrungsaufbau benötigt Zeit. Dies haben nicht zuletzt die arbeitssoziologischen Untersuchungen zum sog. Erfahrungswissen gezeigt (Malsch 1984, Böhle/Milkau 1988; kritisch dazu: Malsch/Mill 1992, S. 17ff.; Mill 1998, S. 29ff.; zuletzt Pfeiffer 2012).

Der Erwerb eines Abschlusses in einer dualen Berufsausbildung sowie eines akademischen Abschlusses in einem Ausbildungsgang führt also dazu, dass Ausbildungszeiten verkürzt werden, Ausbildungsinhalte gekürzt oder komprimiert werden. Insofern kommt der beruflichen Ausbildung ein eher nachgeordneter Stellenwert zu, zumal der spätere betriebliche Einsatz der Absolventen dualer Studiengänge nicht auf Facharbeiterebene vorgesehen ist. Darin ist der Grund zu sehen, dass wir so wenige kritische Stimmen zur Qualität der fachlichen Ausbildung in unserer Untersuchung gefunden haben. Wie es einer der Studierenden ausdrückte: „Und unsere Ausbildung in dem Sinne ist 
auch nicht dahin gerichtet, dass wir halt wirklich einen kompletten Facharbeiter ersetzen." (Fall B, Gruppendiskussion, §72) Die von uns untersuchten Studiengänge bringen keine höherwertigen Kaufleute oder Mechatronikerinnen hervor, sondern Ingenieurinnen und Wirtschaftswissenschaftler, die - ganz im Sinne von Böhle (2010) - von der beruflichen Bildung gelernt haben.

Die Ausbildungsinhalte der Berufe nach Berufsbildungsgesetz (BBiG) und Handwerksordnung (HWO) sind bundesweit curricular festgelegt. Das Spektrum des Wissens und Könnens von z. B. Bürokaufleuten, die in verschiedenen Bundesländern und in verschiedenen Unternehmen ausgebildet worden sind, soll vergleichbar sein - es handelt sich um eine allgemeine, gesellschaftlich anerkannte Handlungskompetenz. Aber im „Lernort Unternehmen“ wird qua Unternehmenskultur auch eine spezifische Kompetenz entwickelt. ${ }^{6}$ Denn nach Abschluss der (zeitlich verkürzten) dualen Ausbildung im dualen Studium sind die dual Studierenden (in sehr unterschiedlichem Ausmaß) in den Unternehmen präsent: So arbeiten sie in den Semesterferien als Fachkräfte in den Unternehmen oder bearbeiten dort für ihr Studium Praxisprojekte. Diese spezifische, unternehmensbezogene Kompetenz wurde von allen Gesprächspartnern, die sie thematisierten (sowohl bei den Studierenden als auch bei den Vertretern von Unternehmen und Kammern), als vorteilhaft für Unternehmen und Absolventen bewertet. Da das zentrale Motiv der Unternehmen, sich an dualen Studiengängen zu beteiligen, die Fachkräftegewinnung und -bindung ist, ist die positive Bewertung der Entwicklung dieser unternehmensspezifischen Kompetenz durch die Personalverantwortlichen nicht erstaunlich - zumal sich die erworbenen Kompetenzen nicht ohne Weiteres in andere Unternehmen transferieren lassen. Die Studierenden, die sich vor allem in der Konkurrenz zu anderen Wirtschaftswissenschaftlern oder Ingenieuren mit Bachelorabschluss sehen, reklamieren hier einen Kompetenzvorsprung gegenüber diesen Hochschulabsolventen.

Unabhängig davon, ob die Handlungskompetenz der dual Studierenden tatsächlich dem einer gelernten Kauffrau oder eines gelernten Facharbeiters entspricht, tragen die Absolventen der ausbildungsintegrierenden dualen Studiengänge einen entsprechenden Titel, der durch Prüfungen bei der Industrie- und Handelskammer (IHK) oder - selten! - bei der Handwerkskammer $(\mathrm{HWK})^{7}$ beglaubigt und anerkannt worden ist. Die zentrale Funktion von Titeln sieht Bourdieu (1983, S. 189f.) darin, dass die Inhaber von Titeln nicht „ständig unter Beweiszwang“ stehen, also ihr Können und Wissen nicht ständig beweisen müssen. Titel identifizieren Titelträger mit einer Kompetenz und unterscheiden die Titelträgerin von den Titellosen, deren Kompetenz fraglich ist. Titel haben damit eine dramaturgische Funktion im Sinne von Goffman (1959). ${ }^{8}$ Das Publikum für das dramaturgische Handeln der Absolventen ausbildungsintegrierender dualer Studiengänge bilden die Facharbeiterinnen oder Kaufleute im Unternehmen einerseits und andererseits die Personalverantwortlichen von Unternehmen.
Für die Facharbeiterinnen oder Kaufleute sind die beruflichen Titel der Absolventen dualer Studiengänge ein Zeichen der Fachkompetenz; sie können darauf setzen, dass die Absolventinnen „einfach dieselben Probleme kennen, die man im Studium nicht unbedingt kennenlernt" (Fall F, Gruppendiskussion, \$126) und deshalb von ihnen „die Erfahrung eines Facharbeiters [nicht!] übertrieben übergangen“ wird (Fall B, Gruppendiskussion, § 106). Im Hinblick auf ihre weitere Karriere etwa in anderen Unternehmen setzten die dual Studierenden in unseren Gruppendiskussionen auf den beruflichen Titel als zusätzliches Signal am Arbeitsmarkt (Spence 1973). Ihre kaufmännischen Titel oder Facharbeitertitel sollen für Personalverantwortliche in Unternehmen einen komparativen Wettbewerbsvorteil der dualen Absolventen gegenüber normalen Absolventen ingenieur- oder wirtschaftswissenschaftlicher Studiengänge darstellen. ${ }^{9}$

\section{Fazit und Ausblick}

Der Trend zur Akademisierung hat die Debatte um die Verdrängung beruflich Gebildeter durch akademisch Ausgebildete bei den Führungskräften angeheizt. Diese Debatte wird auch für das duale Studium in einigen wenigen Untersuchungen geführt. Mit unserer Untersuchung konnte die These, dass die Absolventen ausbildungsintegrierender dualer Studiengänge den Facharbeiter mit Aufstiegsfortbildung (Meister, Techniker usw.) ersetzen, nicht belegt werden. Vielmehr führt sie zur Hypothese, dass in Unternehmen, die dual ausgebildete Ingenieure rekrutieren, diese komplementär zu den Facharbeitern mit Aufstiegsfortbildung eingesetzt werden. Im Feld der dualen Betriebswirte dagegen herrscht teilweise eine offene Konkurrenzsituation zwischen den einfachen Bachelorabsolventen und den Kaufleuten, die Aufstiegsfortbildungsabschlüsse vorweisen können. Insbesondere im Bankenbereich führt, so unsere Hypothese, die weniger ausgebaute Differenzierung der Karrierepfade zur Konkurrenz.

Was allerdings den Absolventen ausbildungsintegrierender dualer Studiengänge fehlt, ist der Aufbau beruflicher Erfahrung im Medium des Fachberufes - als Mechatronikerin, als Industriekaufmann etc. Die Nachrangigkeit der Berufs-

6 Wir lehnen uns hier an die Unterscheidung von allgemeinem und spezifischem Humankapital an (vgl. Becker 1992, S. $44 f$.).

7 In einem unserer 20 zufällig ausgewählten Fallstudien war eine Handwerkskammer beteiligt.

8 Siehe den Begriff des dramaturgischen Handelns im Anschluss an Goffman u. a. bei Habermas (1988, S. 128).

9 Wir beschränken uns hier auf diese zwei "Zielgruppen”, um das Argument zu verdeutlichen. 
ausbildung im dualen Studium wird durch die starke Verkürzung der Ausbildungszeiten und die Kompression des Lernstoffs deutlich. Die Bedeutung der beruflichen Kompetenz im späteren Einsatz der Absolventen in den Unternehmen bleibt aber weiter zu untersuchen.

Wir haben in unserer Untersuchung das ausbildungsintegrierende duale Studium betrachtet. Durch die Befragung von Unternehmen, die in dualen Studiengängen kooperieren, konnten wir empirisch begründete Hypothesen über den zukünftigen Einsatz der Absolventen dieser Studiengänge aufstellen. Es wäre aus unserer Sicht an der Zeit, diese Hypothesen in einer größeren Verbleibsstudie von Absolventen zu überprüfen, die mit einer Erhebung von Einsatzmustern von Absolventen in Unternehmen kombiniert werden müsste. Wie sind die Absolventen in Unternehmen positioniert und gratifiziert worden? Welche Kompetenzen konnten sie wie nutzen? Ist ein Verdrängungswettbewerb zu beobachten? Gibt es signifikante Unterschiede zwischen dem kaufmännischen Feld und dem Feld der technischen Berufe? Die Antworten auf diese Fragen münden in eine empirisch begründete Theorie des Einsatzes der Absolventen von dualen Studiengängen.

\section{LITERATUR}

Baethge, M./Solga, H./Wieck, M. (2007): Berufsbildung im Umbruch. Signale eines überflüssigen Aufbruchs: Friedrich-Ebert-Stiftung, Berlin

Becker, G. S. (1992): The Economic Way of Looking at Life, Nobel Lecture.

Böhle, F. (2010): Kann die höhere Bildung von der beruflichen Bildung lernen? Die Verbindung von institutionalisiertem Lernen und praktischem Tun eröffnet neue Lernfelder und -orte, in: Berufsbildung in Wissenschaft und Praxis (BWP) 39 (2), S. $6-9$

Böhle, F./Milkau, B. (1988): Vom Handrad zum Bildschirm. Eine Untersuchung zur sinnlichen Erfahrung im Arbeitsprozeß, Frankfurt,a. M./New York

Bosch, G. (2012): Gefährdung der Wettbewerbsfähigkeit durch zu wenige Akademiker: echte oder gefühlte Akademikerlücke?, in: Kuda, E./Strauß, J./ Spöttl, G./Kaßebaum, B.: Akademisierung der Arbeitswelt. Zur Zukunft der beruflichen Bildung, Hamburg, S. 20-35

Bourdieu, P. (1983): Ökonomisches Kapital, kulturelles Kapital, soziales Kapital, in: Kreckel, R. (Hrsg.): Soziale Ungleichheiten, Göttingen, S.183-198 Bundesministerium für Bildung und Forschung (BMBF) (2012): Berufsbildungsbericht 2012, Berlin

Bundesministerium für Bildung und Forschung (BMBF) (2013): Berufsbildungsbericht 2013, Berlin

Drexel, I. (2012): Gesellschaftliche und politische Folgen von Akademisierung, in: Kuda et al., a. a. O., S. 36-51

Franz, C./Voss-Dahm, D. (2011): Ohne Studium (k)eine Führungsposition? Nach wie vor starke Bedeutung von beruflichen Bildungsabschlüssen bei Führungskräften in der Privatwirtschaft, IAQ-Report 2011-02, Duisburg Goffman, E. (1959): The Presentation of Self in Everyday Life, New York/ Toronto

Graf, L. (2012): Wachstum in der Nische. Mit Dualen Studiengängen entstehen Hybride von Berufs- und Hochschulbildung, in: WZB-Mitteilungen (138),

S. $49-52$

Habermas, J. (1988): Theorie des kommunikativen Handelns. Erster Band, Frankfurt a. M.

Heidemann, W. (Hrsg.) (2011): Duale Studiengänge in Unternehmen:

Hans-Böckler-Stiftung, Arbeitspapier (236), Düsseldorf

Helmrich, R./Zika, G./Kalinowski, M./Wolter, M. I. (2012): Engpässe auf dem Arbeitsmarkt: Geändertes Bildungs- und Erwerbsverhalten mildert Fachkräftemangel - Neue Ergebnisse der BIBB-IAB-Qualifikations- und Berufsfeldprojektionen bis zum Jahr 2030, BIBB-Report 18/2012, Bonn

Hollmann, C./Schmidt, J./Werner, D. (2010): Verdrängt der Bachelor duale Ausund Fortbildungsberufe?, in: Berufsbildung in Wissenschaft und Praxis 39 (2), S. $18-22$

Kloas, P.-W. (2007): Zusatzqualifikationen und duale Studiengänge im Handwerk, in: Beitrag für Handwerk Magazin, Beruf \& Bildung 11/2007, S. 1-5 Klumpp, M./Kriebel, K./Beschorner, H./Buschfeld, D./Dilger, B./Diart, M. (2010): Berufswertigkeit konkret. Wissenschaftlicher Abschlussbericht, Köln Klumpp, M./Rybnikova, I. (2010): Differenzierte Studienformen - Eine empirische Forschungserhebung in Deutschland, Bielefeld
Krone, S./Mill, U. (2012): Dual studieren im Blick: Das ausbildungsintegrierende Studium aus der Perspektive der Studierenden, IAQ-Report 2012-3, Duisburg Kruse, W./Strauß, J./Braun, F./Müller, M. (2009): Rahmenbedingungen der Weiterentwicklung des Dualen Systems beruflicher Bildung: Hans-BöcklerStiftung, Arbeitspapier (168), Düsseldorf

Kuda, E./Strauß, J. (Hrsg.) (2010a): Akademisierung von Betrieben - Facharbeiter/innen ein Auslaufmodell?, Frankfurt a. M.

Kuda, E./Strauß, J. (Hrsg.) (2010b): Beruflich-betriebliche Bildung - Sind die Handlungs- und Sozialisationsmodelle überholt?, Frankfurt a. M.

Kuda, E./Strauß, J. (Hrsg.) (2011): Beruflich-betriebliche Bildung - noch prägend für Arbeit und Gesellschaft?, Frankfurt a.M.

Kuda, E./Strauß, J./Spöttl, G./Kaßebaum, B. (Hrsg.) (2012): Akademisierung der Arbeitswelt. Zur Zukunft der beruflichen Bildung, Hamburg

Kupfer, F./Kolter, C./KöhImann-Eckel, C. (2012): Analyse und Systematisierung dualer Studiengänge an Hochschulen, Bonn

Malsch, T. (1984): Erfahrungswissen versus Planungswissen. Facharbeiterkompetenz und informationstechnologische Kontrolle am Beispiel der betrieblichen Instandhaltung, in: Jürgens, U./Naschold, F. (Hrsg.): Arbeitspolitik. Materialien zum Zusammenhang von politischer Macht, Kontrolle und betrieblicher Organisation der Arbeit, Opladen, S. 231-251

Malsch, T./Mill, U. (Hrsg.) (1992): ArBYTE. Modernisierung der Industriesoziologie?, Berlin

Mill, U. (1998): Technik und Zeichen. Über semiotische Aktivität im technischen Kotext, Baden-Baden

Organisation for Economic Co-operation and Development (OECD) (2012): Bildung auf einen Blick - OECD-Indikatoren, Bielefeld

Organisation for Economic Co-operation and Development (OECD) (2013): Bildung auf einen Blick - OECD-Indikatoren, Bielefeld

Pfeiffer, S. (2012): Wissenschaftliches Wissen und Erfahrungswissen, ihre Bedeutung in innovativen Unternehmen und was das mit (beruflicher) Bildung zu tun hat, in: Kuda et al., a. a. O., S. 203-219

Purz, S. (2011): Duale Studiengänge als Instrument der Nachwuchssicherung Hochqualifizierter, Frankfurt a. M.

Spence, M. (1973): Job Market Signaling, in: The Quarterly Journal of Economics 87 (3), S. 355-374

Statistisches Bundesamt (2012): Bildung und Kultur. Schnellmeldungsergebnisse der Hochschulstatistik zu Studierenden und Studienanfänger/-innen vorläufige Ergebnisse, Wiesbaden

Statistisches Bundesamt (2013): Hochschulen auf einen Blick, Wiesbaden Strauß, J./Kuda, E. (Hrsg.) (2010): Universitäre und berufliche Bildung Annäherung oder Entfernung?, Frankfurt a. M.

Weigel, T./Hippach-Schneider, U./Gonon, P. (2011): Qualität von Bildungsabschlüssen aus Unternehmenssicht - eine kompetenzorientierte Analyse, bwp@ Ausgabe Nr. 21, Dezember

Werner, D./Hollmann, C./Schmidt, J. (2008): Wie entwickeln sich angesichts des Strukturwandels zur Wissensgesellschaft und der Einführung der Bachelorstudiengänge die Chancen für duale Ausbildungsberufe und das duale System?: Institut der deutschen Wirtschaft, Köln

Wünsche, T. (2011): Betriebliche Qualifikationsbedarfsdeckung im Fachkräftebereich wachsender Beschäftigungsfelder, unveröff. Abschlussbericht, Bonn

\section{AUTOREN}

SIRIKIT KRONE, Dr., ist Wissenschaftliche Mitarbeiterin der Forschungsabteilung Bildung und Erziehung im Strukturwandel am Institut Arbeit und Qualifikation (IAQ) an der Universität Duisburg-Essen. Arbeitsschwerpunkte: Übergänge im Bildungssystem, Entwicklungen im tertiären Bildungssektor.

sirikit.krone@uni-due.de

ULRICH MILL, Dr., Freiberufler und zur Zeit Gastwissenschaftler am IAQ. Aktuelle Arbeitsschwerpunkte: Bildungssegmentation, Anerkennung beruflicher Abschlüsse.

ulrich.mill@uni-due.de 